\title{
EVALUACIÓN DEL PREJUICIO RACIAL IMPLÍ- CITO (IAT): CASO REPÚBLICA DOMINICANA
}

\section{Evaluation of Implicit Racial Prejudice (IAT): Case Dominican Republic}

\section{Yesilernis L. Peña, Ph.D.1}

Resumen: La presente investigación tiene como propósito fundamental determinar la veracidad de la teoría de democracia racial versus la existencia de racismo en Latinoamérica a través de la medición de perjuicio racial implícito. También procura medir el nivel de prejuicio racial en la República Dominicana mediante métodos implícitos, así como comparar los niveles de prejuicio racial por grupo racial y comparar los hallazgos de perjuicio racial implícito latinoamericanos con los hallazgos en países anglosajones. Con los hallazgos analizados en el grupo particular que ha sido estudiado puede inferirse que hay una mezcla de resultados en términos de la democracia racial percibida y el racismo que otros autores han encontrado en la República Dominicana.

Tanto a nivel de subconsciente (actitud implícita) como consciente reportado (actitud explícita) los participantes en presentan prejuicio implícito en general a favor de los negros y en contra de los blancos, es decir, idealismo de superioridad africana, de forma explícita las mujeres presentan un menor idealismo de superioridad africana que los hombres. El tema reviste una capital importancia social dado que se esperaría todo lo contrario y señala lineamientos de investigación para entender la convivencia interracial y la equidad racial en la República Dominicana.

Palabras clave: Racismo, democracia racial, prejuicio racial implícito, pigmentocracia.

1 Profesora Investigadora del INTEC. 
Abstract: This investigation has as main purpose to determine the veracity of the theory of Racial Democracy versus the existence of racism in Latin America through the measurement of implicit racial prejudice. Also seeks to measure the level of racial prejudice in the Dominican Republic using implicit methods and compare the levels of racial prejudice by racial group and compare the findings of Latin American implicit racial prejudice with findings in Anglo-Saxon countries. With the findings discussed in the particular group that has been studied we can infer that there are mix findings in terms of perceived racial democracy and the racism found by other authors in the Dominican Republic.

Both in terms of unconscious (implicit attitude) as a conscious reported (attitude explicit) participants have implicit prejudice in general in favor of blacks and against whites, i.e. idealism of African superiority. Explicitly, women have less idealism of African superiority than men. The issue is of paramount social importance because the opposite would be expected and sets guidelines for future research to understand the interracial coexistence and racial equity in the Dominican Republic.

Keywords: Racism, racial democracy, prejudice, implicit racism, pigmentocracy.

\section{Introducción}

El debate académico del prejuicio racial no es nuevo a nivel global, ni en Latinoamérica, en particular. La más antigua tesis del tema de racismo en Latinoamérica, Democracia racial (ver Freyre, 1951), mantiene que el prejuicio racial no existe en dicha región. Mientras que la tesis de El mito de la democracia racial indica que la idea de democracia racial fue creada para asegurarse de que los países latinoamericanos mantuvieran la jerarquía racial (Degler, 1986). 
En particular, una teoría que sale de la ciencia de la psicología, la teoría de Dominancia social ("Social Dominance"; J. Sidanius, 1993), indica que el prejuicio racial existe en todas las culturas y que siempre la raza dominante es la que posee este prejuicio en contra de la raza denominada como "inferior". Sin embargo, cuando parte de este mismo grupo de autores intentaron confirmar esta tesis en Latinoamérica, usando el caso de la República Dominicana (Sidanius, Peña \& Sawyer, 2001), encontraron resultados mixtos; por un lado obtuvieron evidencia de una fuerte evidencia de "pigmentocracia," o jerarquía social basada en el color de la piel, pero por otro lado, mostraron que no había asociación positiva con racismo antinegro.

Estos hallazgos contradictorios demandan una evaluación más cercana a la detección de prejuicio racial psicológico en países latinoamericanos y en particular, en República Dominicana. La técnica de medición de actitudes implícitas ("Implicit Attitude Test"; (IAT), ha sido utilizada con frecuencia en países como los EE. UU y Europa para evidenciar el prejuicio racial psicológico existente, ya que elimina el sesgo de tratar de proyectar imparcialidad vía los métodos tradicionales de escalas y cuestionarios (deseabilidad social; "social desirability"). Sin embargo, la medición de actitudes implícitas ha sido poco usada en Latinoamérica.

En esta investigación ha sido utilizada la metodología de medición implícita para medir prejuicio racial en la República Dominicana a un nivel psicológico subconsciente, nivel que dicta del pensar, de las evaluaciones y acciones de los individuos en una sociedad. Se espera que se encuentren manifestaciones de prejuicio racial implícito entre los dominicanos, utilizando una muestra de estudiantes de la universidad de INTEC, en Santo Domingo.

Hemos encontrado que tanto a nivel de subconsciente (actitud implícita) como consciente reportado (actitud explícita) los participantes en el estudio presentan prejuicio implícito en general a favor de los negros y en contra de los blancos, es decir, idealismo 
de superioridad africana. Esto constituye un hallazgo extremadamente importante ya que puede señalar que aunque el "negro" pudiera aun ser discriminado, el hecho de ser mayoría, lo destaca perceptualmente como que debe ser mejor o superior. Como nota, encontramos que de forma explícita las mujeres presentan un menor idealismo de superioridad africana que los hombres. Esto representa un aporte de gran valor a las investigaciones de racismo en Latinoamérica ya que hasta el momento se ha encontrado que el mismo "negro" se discrimina a sí mismo (Sidanius, Peña \& Sawyer, 2001) y esta investigación indica lo contrario.

Lo anterior representa también un avance hacia la unión de los pueblos de descendencia africana para un mejoramiento de la situación racial en la República Dominicana y en Latino américa. Sin embargo, este estudio fue llevado a cabo con estudiantes universitarios y se debería expandir esta investigación a la población de la República Dominicana mediante un próximo estudio para reforzar y entender si se replica en el resto del país.

\section{Antecedentes y descripción del problema de investigación}

La tesis conocida como la teoría de la democracia racial sostiene que, en comparación con Estados Unidos, las naciones de América latina son en gran parte libre del prejuicio racial feroz que ha caracterizado las relaciones raciales en los Estados Unidos durante la mayor parte del siglos XIX y XX (véase a Degler, 1986; Freyre, 1951; Hoetink, 1967; Pierson, 1942; Tannenbaum, 1947).

Los teóricos de la democracia racial basan estas conclusiones en el hecho de que, en comparación con América del Norte, las naciones de Latinoamérica muestran una marcada ausencia de resultados negativos posterior a la esclavitud (ausencia de racismo institucionalizado, como por ejemplo, la ausencia segregación y leyes (véase Sidanius, 1993), ausencia general de violencia grupal basada 
en la raza (por ejemplo, los linchamientos y otros crímenes de odio basados en la raza como ocurrieron en los EE. UU. las protestas masivas en contra del racismo y un sorprendente alto índice de mestizaje.

La desigualdad racial es aún perceptible en las sociedades latinoamericanas, esta desigualdad se debe casi exclusivamente a los efectos residuales del pasado racista de asignación de recursos y no a los efectos de los prejuicios raciales en curso.

Los teóricos de la democracia racial atribuyen igualdad racial de los países latinoamericanos basados en tres factores principales. En primer lugar, a diferencia de los colonizadores europeos de América del Norte, los conquistadores ibéricos de Latinoamérica tuvieron la experiencia de vivir bajo la hegemonía política y social de los moros, personas de piel oscura, por casi 800 años. Después de haber experimentado estos conquistadores de piel oscura como sus políticas y culturales "superiores", los colonos ibéricos no podrían entonces considerar los esclavos de piel oscura de África y de la India como subhumanos con el mismo grado de celeridad que los colonos norteamericanos encontraron posible. En segundo lugar, contrariamente a las doctrinas puritanas de protestantismo norteamericano, el catolicismo considera a los nativos e incluso los esclavos africanos, como personas con almas e igualmente amados por Dios; por lo tanto, los conquistadores católicos eran menos reacios a entremezclarse con ellos. Por último, en contraste con los colonos de América del Norte, los primeros colonos de América Latina no se aventuraron al Nuevo Mundo con familias intactas. Como resultado, los colonizadores ibéricos establecieron relaciones sexuales y emocionales con las esclavas indias y africanas con bastante rapidez, creando una actitud más positiva hacia las personas de ascendencia africana y que resulta en las altas tasas de mestizaje que vemos hoy en día en América Latina (Degler, 1986; Freyre, 1951). 
Derivado de lo anterior, se sostiene que los movimientos sociales de base amplia, como el Movimiento de los Derechos Civiles en Estados Unidos (1960), no eran necesarias debido a la naturaleza más igualitaria de las relaciones raciales en América Latina. Sin embargo, la tesis de la democracia racial en América Latina ha sido objeto de crecientes ataques por los teóricos críticos de la raza (Guimaraes, 2002). Dado el hecho de que los blancos disfrutan de ventajas apreciables sobre los negros en áreas como salud, educación, ingresos, profesión, estatus y poder político en muchos de los países, incluyendo Brasil, México, Colombia, Venezuela, Nicaragua, Panamá, Costa Rica, Perú, Bolivia y la República Dominicana (véase el Minority Rights Group, 1995; Wade, 1997).

Los teóricos críticos de la raza sostienen que la única cosa excepcional sobre las relaciones raciales latinas es el éxito con el que los países de América Latina han promulgado el mito más que la realidad de la igualdad racial (Hanchard, 1994; Marx, 1998; Nobles, 2000). Los críticos argumentan que los defensores de la tesis de la democracia racial cometen el error de ver las relaciones raciales en América Latina a través del prisma de las dinámicas raciales que se encuentran en los Estados Unidos. Como resultado, los teóricos de la democracia racial han sido insensibles a las distintas formas de exclusión racial que se practican en Latinoamérica. Por ejemplo, los países latinoamericanos tienen una historia de privilegios y leyes que dan puestos de trabajo, la tierra, exenciones de impuestos y otros beneficios a los inmigrantes europeos y asiáticos en un esfuerzo por disminuir la porción relativa de los negros en las sociedades latinas a través del "blanqueamiento" (Marx, 1998; Moya-Pons, 2000; Nobles, 2000; Wade, 1997).

En lugar de tratar de eliminar a las personas de ascendencia africana a través de la violencia informal (por ejemplo, los pogromos), altas tasas de encarcelamiento o ejecución, las naciones de América Latina han optado por atenuar la influencia africana a través del proceso de dilución (Nobles, 2000). Los críticos sostienen que el objetivo de ambas regiones, American del Norte y América Latina, 
sigue siendo, en última instancia, la misma: hegemonía blanca. Por lo tanto, la democracia racial es vista como un mito que funciona para mantener la misma cosa que niega: opresión racial (Twine, 1998).

Entre otras vías, la creencia de la democracia racial se logra mediante la instalación de identificación y lealtad a la nación en su conjunto y no a grupos raciales específicos, lo cual impide el desarrollo de la movilización social basada en grupos, lo que es necesario para hacer frente a los agravios sociales reales (Hanchard, 1994; Marx, 1998).

Además, sostienen que, en la era post-derechos Civiles en Estados Unidos, con el advenimiento de las políticas más conscientes a la raza y el reconocimiento de desventajas formales, las comparaciones de las relaciones raciales en América Latina con Estados Unidos no siempre favorecen a Latinoamérica (Degler, 1986; Hanchard, 1994; Marx, 1998). Ellos argumentan que la falta de movimientos sociales basadas en la raza y la negación de la desigualdad racial han creado un espacio discursivo donde florece el racismo abierto, pero se niega debido al mito de la democracia racial. En contraste, los Estados Unidos, todavía luchan por superar el legado de exclusión racial, por lo que han tenido que abordar conscientemente la falta de incorporación de los negros en todos los ámbitos de la vida política, social y económica.

Sin embargo, es posible que el argumento de que las disparidades económicas y sociales que se evidencian en Latinoamérica entre los negros y los blancos no son más que el legado persistente de la esclavitud y que no tiene nada que ver con el curso racial y el prejuicio. Además, incluso si el prejuicio racial sigue existiendo en Latinoamérica, aún es posible que una versión más débil de la tesis racial en torno a la democracia siga siendo válida. Esto quiere decir que, a pesar de los prejuicios aún existentes, el racismo podría no obstante ser significativamente más débil en América Latina que en América del Norte. 
El estudio de Sidanius, Peña \& Sawyer (2001) intentó comparar estas teorías directamente. El estudio exploró la naturaleza de la jerarquía racial y la conexión entre la identidad racial y el patriotismo de República Dominicana mediante un cuestionario aplicado a una muestra en situ en la República Dominicana. Los análisis compararon las expectativas contradictorias de la teoría de la "democracia racial" tesis y el mito de la democracia racial, utilizando las hipótesis de la teoría de "dominancia social" (Sidanius, 1993).

Los resultados mostraron que a pesar del alto nivel de endogamia racial en la República Dominicana, existe una fuerte evidencia de una "pigmentocracia," o jerarquía social basada en el color de la piel. Por otra parte, a pesar de una ligera tendencia a la gente a dar un poco más altas calificaciones de estado a su propia categoría "racial" de la que fue dado a ellos por los miembros de otras categorías "raciales", esta pigmentocracia era altamente consensuada a través de la jerarquía racial. Estos resultados fueron más consistentes con las expectativas de la teoría de la dominación social (mito de democracia racial) que de "democracia racial".

En contraste con análisis similares tanto en los Estados Unidos e Israel, estos hallazgos dominicanos no mostraron ninguna evidencia de que los miembros de diferentes categorías "raciales" tenían diferentes niveles de apego patriótico a la nación. También en contraste con los resultados norteamericanos recientes, no había pruebas de que el patriotismo dominicano se asoció positivamente con el racismo anti-negro, orientación de dominancia social, el afecto negativo hacia otros grupos raciales, ni el etnocentrismo, independientemente de la categoría "racial" al cual los participantes pertenecían. Estos últimos resultados son más consistentes con la tesis de la democracia racial." Al final, este importante estudio realizado en la República Dominicana, no pudo ni confirmar ni contradecir contundentemente ninguna de las dos teorías.

Para intentar resolver esta controversia debemos utilizar instrumentos que nos permitan llegar a la base primaria de la psicología 
humana, la parte del subconsciente. Esta es la parte de la mente del ser humano que dicta muchas de nuestras acciones, incluyendo el racismo (Chen \& Bargh, 1997). Aunque el uso de medidas explícitas de actitud racial ha sido el método estándar de evaluación de las actitudes raciales de los últimos 60 años, esta técnica puede no ser el método más apropiado para la evaluación de los prejuicios raciales en algunas culturas.

Conviene recordar que las medidas explícitas de prejuicio racial sufren el mayor nivel de la deseabilidad social y la auto-presentación del sesgo (Carver \& Katz, 1978). Cuando nos enfrentamos a cuestiones raciales explícitas, altamente sensibles y potencialmente inflamatorios, los participantes se sienten a menudo bajo una considerable presión para proporcionar respuestas políticamente más aceptables que pudieran autorizarse (véase, por ejemplo, Crosby, Bromley y Saxe, 1980; Fazio, Jackson, Dunton \& Williams, 1995; ver también Dovidio \& Fazio, 1992; Wittenbrink, Judd \& Park, 1997). Como resultado, el uso de medidas explícitas ha llevado a los investigadores de las relaciones raciales en Estados Unidos a la conclusión de que el prejuicio racial ha estado en un declive continuo en los últimos 40 años, mientras que las técnicas menos molestas (como los experimentos y estudios a ciega) indican que no hay tal declive (Crosby et al., 1980; Wittenbrink et al., 1997).

El problema de "deseabilidad social" puede ser particularmente problemático en el contexto latinoamericano, donde la promulgación de la democracia racial como ideología dominante ha sido generalizada, incluso entre los latinos (Hanchard, 1994; Marx, 1998). Por otro lado, está la falta de un movimiento coherente para desafiar el discurso y la práctica racista pueden hacer que Latinoamérica, en comparación con los Estados Unidos, tenga menos conciencia sobre el discurso racista y sus prácticas.

Hay menos discursos explícitamente antirracistas e imágenes conscientemente positivas de los negros y menos presión social para 
conformar las actitudes y el discurso a la práctica socialmente deseable. Sin embargo, la brecha entre el prejuicio real y lo que puede ser medido sigue siendo un problema metodológico. Una solución cada vez más popular a este problema de deseabilidad social en la investigación de la actitud racial es utilizar medidas implícitas más que explícitas de perjuicio.

Se han diseñado medidas implícitas de prejuicio racial para establecer índices de las actitudes raciales en formas sutiles que limitan los efectos de las presiones normativas para mostrar respuestas no prejudiciales. Esto se logra con la exploración de los aspectos más automáticos, sobre-entrenados y no conscientes de evaluaciones grupales (Blair, 2001; Bosson, Swann y Pennebaker, 2000; Greenwald, McGhee \& Schwartz, 1998; Wittenbrink et al., 1997).

Desde su desarrollo inicial en la década de 1980 (véase Gaertner y McLaughlin, 1983), las medidas implícitas de actitudes han sido ampliamente utilizadas para evaluar una variedad de actitudes implícitas (automáticas), incluyendo los prejuicios contra los negros, las mujeres y los grupos externos generalizados (Blair, 2001). Además, se ha encontrado que estos índices de los prejuicios implícitos sirven para predecir no solo otras actitudes, pero el comportamiento real, tales como la hostilidad (véase, por ejemplo, Bargh, Chen \& Burrows, 1996; Chen \& Bargh, 1997; Correll, Parque, Judd \& Wittenbrink, 2002; Dijksterhuis \& Bargh, 2001; Payne, 2001).

Conviene señalar que las medidas implícitas de prejuicio comparan generalmente la eficiencia con la que los participantes asocian evaluaciones positivas y negativas con categorías sociales específicas, como las distintas "raza". Así, por ejemplo, comparando el tiempo que les lleva a los participantes emparejar "malo" con "negro" y "bueno" con "Blanco", en comparación con el tiempo necesario para emparejar "bueno" con "Negro" y "malo" con "blanca", uno puede determinar qué conjunto de emparejamientos llega más fácilmente a la mente. Si el emparejamiento de "bueno" 
con blanco y "malo" con negro son cognitivamente más accesible que la asociación opuesta, entonces puede decirse que los participantes manifiestan prejuicios raciales contra los negros respecto a los blancos.

En una cultura con estereotipos raciales y una larga historia de racismo, como los Estados Unidos, se ha encontrado consistentemente que a los blancos les resulta más fácil asociar negro con "malo" y blanco con "bueno" que a la inversa (véanse las revisiones de Blair de 2001, así como Greenwald \& Nosek, 2001; también véase Seise \& Zerbes, 2001; Kuehnen et al., 2001; McConnell \& Leibold, 2001). Sin embargo, estas medidas no son típicamente usadas en el contexto latinoamericano. Al utilizarla pudiéramos evaluar el grado en que el aprendizaje social podría afectar asociaciones implícitas sobre la raza.

Si se comparan los hallazgos latinoamericanos contra los hallazgos en países anglosajones, pudiéramos entender temas relevantes, como el aprendizaje a raíz del discurso político de la nación. Este análisis comparativo nos permitiría examinar la fuerza que tiene o no el discurso de la democracia racial en Latino América, para determinar si ha permeado los niveles subconscientes. Para explorar estas áreas este estudio utilizará métodos de medición del prejuicio racial implícito en distintos grupos de color de piel ("raza") en República Dominicana.

\section{Hipótesis}

Hay dos tesis contradictorias existentes en la literatura del prejuicio racial en Latinoamérica: democracia racial y mito de la democracia Racial. Si la versión de la tesis de "democracia racial" es correcta, entonces no deberíamos encontrar ninguna clase de prejuicio racial implícito en la República Dominicana. Si la tesis del mito de la democracia Racial es correcta, se debería encontrar prejuicio racial implícito (nivel que afecta las actitudes que poseemos y acciones que realizamos). 
Es crucial determinar la existencia de prejuicio racial desde la perspectiva psicológica y subconsciente en Latinoamérica y, en particular, en la República Dominicana, para así poder plantear acciones a tomar para combatir sus efectos desde un punto psicológico en caso de mostrar la existencia de dicho prejuicio. Ya que ha habido mucha controversia con este tema y los hallazgos son contradictorios, este estudio busca la manera de poder medir y evidenciar la manifestación subconsciente de dicho prejuicio.

\subsection{Participantes}

Participaron en el estudio unos 154 voluntarios de ambos sexos (H: 78; M: 76), con edades comprendidas entre los 17 y 28 años de edad, de diferentes razas, tonalidades de piel, nivel académico, entre estudiantes universitarios de grado y postgrado, así como de diversas carreras en la ciudad de Santo Domingo. Estos individuos fueron seleccionados con la técnica de muestreo por conveniencia no probabilística, fueron reclutados en el recinto del Instituto Tecnológico de Santo Domingo (INTEC), debido a la sensibilidad de este tema, el presupuesto y la particularidad de los materiales a utilizar que requieren mesas, sillas y equipos informáticos con internet para realizar la investigación implícita.

\subsection{Materiales y procedimientos}

\section{Prejuicio implícito}

Fueron utilizadas 6 computadoras tipo laptops con internet y los softwares Windows 7 y Cantasia Studio versión 6; para la captura de la información digitada en la plataforma web de la universidad de Harvard para el análisis experimental de Actitud Implícita, disponible en el portal: https://implicit.harvard.edu/implicit/.

La tabla N. ${ }^{\circ} 1$ describe la secuencia de tareas que constituyen las medidas de IAT en esta investigación. 
Tabla N. ${ }^{\circ} 1$

Secuencia de los bloques y estímulos para el estudio de prejuicio implícito

\begin{tabular}{|c|c|c|c|c|}
\hline $\begin{array}{l}\text { Secuencia } \\
\text { (Bloque) }\end{array}$ & 3 & 4 & 6 & 7 \\
\hline $\begin{array}{l}\text { Descripción de } \\
\text { tarea }\end{array}$ & $\begin{array}{l}\text { Inicial } \\
\text { Concepto } \\
\text { objetivo - } \\
\text { Concepto de } \\
\text { discriminación }\end{array}$ & $\begin{array}{l}\text { Tarea Inicial } \\
\text { Combinada } \\
\text { (Palabras positi- } \\
\text { vas y negativas) }\end{array}$ & $\begin{array}{l}\text { Inversa de } \\
\text { Concepto } \\
\text { objetivo - } \\
\text { Concepto de } \\
\text { discriminación }\end{array}$ & $\begin{array}{l}\text { Inversa de } \\
\text { Combinada } \\
\text { (Palabras positi- } \\
\text { vas y negativas) }\end{array}$ \\
\hline $\begin{array}{l}\text { Instrucciones } \\
\text { de la tarea. }\end{array}$ & $\begin{array}{l}+ \text { Negro } \\
\text { Blanco }+\end{array}$ & $\begin{array}{l}+ \text { Negro } \\
+ \text { Positiva } \\
\text { Blanco }+ \\
\text { Negativa }+\end{array}$ & $\begin{array}{l}\text { Negro }+ \\
+ \text { Blanco }\end{array}$ & $\begin{array}{l}\text { Negro }+ \\
+ \text { Positiva } \\
+ \text { Blanco } \\
\text { Negativo + }\end{array}$ \\
\hline $\begin{array}{l}\text { Estímulo de la } \\
\text { Muestra }\end{array}$ & $\begin{array}{l}\text { Fotografías de } \\
\text { personas de } \\
\text { color y blancas. } \\
\text { Blanco }+ \\
+ \text { Negro } \\
+ \text { Negro } \\
\text { Blanco }+ \\
+ \text { Negro } \\
\text { Blanco }+ \\
\text { Blanco }+ \\
+ \text { Negro }\end{array}$ & $\begin{array}{l}\text { Fotografías de } \\
\text { personas de } \\
\text { color y blancas } \\
\text { en combinación } \\
\text { con palabras } \\
\text { negativas y } \\
\text { positivas. } \\
\text { + Blanca } \\
\text { + Positivo } \\
\text { Blanco + } \\
\text { Negativo + } \\
\text { Negro + } \\
\text { + Positivo } \\
\text { + Negro } \\
\text { Negativo + }\end{array}$ & $\begin{array}{l}\text { Fotografías de } \\
\text { personas de } \\
\text { color y blancas. } \\
+ \text { Blanco } \\
\text { Negro }+ \\
\text { Negro + } \\
+ \text { Blanco } \\
\text { Negro + } \\
+ \text { Blanco } \\
+ \text { Blanco } \\
\text { Negro + }\end{array}$ & $\begin{array}{l}\text { Fotografías de } \\
\text { personas de } \\
\text { color y blancas } \\
\text { en combinación } \\
\text { con palabras } \\
\text { negativas y } \\
\text { positivas. } \\
\text { Blanca }+ \\
\text { Positivo + } \\
+ \text { Blanco } \\
\text { + Negativo } \\
\text { + Negro } \\
\text { Positivo + } \\
\text { Negro + } \\
\text { + Negativo }\end{array}$ \\
\hline
\end{tabular}

\subsection{Fases del procedimiento}

El tiempo total para completar la medición por participante fue de 45 minutos (entre la aplicación el cuestionario demográfico, las explicaciones del investigador a cargo del juego, las preguntas de los participantes y las siguientes fases). 
- Primera fase: A los participantes se les asignó una PC con la aplicación de IAT descrita anteriormente y se les explicará el mecanismo de funcionamiento del juego, así como el uso y la interacción de las teclas usadas para seleccionar.

- El procedimiento continuó con la introducción del objetivoconcepto de discriminación (Véase tabla N. ${ }^{\circ} 1$ ), esta y las subsecuentes discriminaciones fueron realizadas asignando una categoría a una respuesta en la mano izquierda (letra E) y la otra respuesta con la mano derecha (Letra I). El segundo paso consistió en la introducción de la dimensión de los estímulos combinados entre raza y palabras positivas-negativas, los cuales se intercambian según el esquema que presentamos en la tabla N. ${ }^{\circ} 1$.

- Este estudio contiene además una batería con seis afirmaciones relativas a dominancia racial y estereotipos, así como un termómetro sobre percepción racial. Para realizar contrastes adicionales fueron adicionadas preguntas a los evaluadores sobre la raza y color de piel de los participantes con el fin de observar algún efecto adicional.

- Cálculo de prejuicio racial implícita: El prejuicio racial implícito se calcula restando el tiempo (en microsegundos) necesario para ordenar los emparejamientos estereotipados (por ejemplo, Malo-Negro y Bueno-Blanco) del tiempo necesario para ordenar los emparejamientos contra-estereotípico (es decir, Bueno-Negro y Blanco-Malo), usando el segundo conjunto de tiempos de la segunda ronda de testeo. La diferencia de resultados positivos indican asociaciones estereotipadas más fáciles que los prejuicios raciales contra-estereotípico y por lo tanto mayor prejuicio implícito contra los negros respecto a los blancos (confirmando la tesis del mito de la democracia racial); puntuaciones de diferencia cero indican que ambas asociaciones eran igualmente accesibles y se interpretan como 
que no muestran prejuicios raciales de ninguna índole (confirmando la teoría de democracia racial); la diferencia de resultados negativos se interpreta como prejuicio implícito en favor de los negros y en contra de los blancos (esto sería como idealismo de superioridad africana).

Los datos fueron manejados y analizados con el paquete informático IBM SPSS versión 19.

\section{ANÁLISIS EXPLORATORIO}

Los datos trabajados contienen los tiempos de respuesta en cada uno de los bloques en el estudio (3, 4, 6 y 7). En el registro de los tiempos no fueron detectados valores en blanco o perdidos. Fueron excluidos del análisis aquellos casos en los que el tiempo de respuesta de cada bloque superó los 60,000 milisegundos, igual procedimiento fue realizado con los datos inferiores a $10 \mathrm{~ms}$. Luego de este procedimiento el tamaño de la muestra se redujo a 115 casos.

Se realizó una transformación a logaritmos naturales a las variables conteniendo los tiempos de respuesta de los bloques en estudio, esto con la finalidad de ajustar los datos al supuesto de normalidad para el uso de modelos de análisis de varianza (ANOVA) y de regresión.

En la batería que mide dominancia y prejuicio racial de las preguntas (P9 a la P14), las escalas fueron recodificadas, debido a que presentaban un orden de mayor y menor en la escala ordinal; esta fue invertida de menor a mayor.

\subsection{Estadísticas y modelo de actitud implícita (IAT)}

Existe una diferencia de medias entre el bloque 6 (concepto inverso objetivo-discriminación) y el bloque 3 (concepto objetivo - discriminación) de aprox. -4,939ms, con una alta significancia 
estadística; sin embargo aunque se nota una diferencia de $-895 \mathrm{~ms}$ bloque 4 y el 7 ((concepto objetivo + estímulo - discriminación + estímulo) no tiene significancia estadística. Para tomar una decisión sobre esta paradoja de significación estadística y debido a que la diferencia de ambos experimentos tienen una correlación positiva (0.23 y una significancia $\mathrm{p}=0.017)$, fue realizado un análisis de diferencias entre la diferencia de medias de (B6-B3) y (B7-B4) las cuales presentan un valor negativo con un alto nivel de significancia estadística. Con los resultados de esta muestra puede evidenciarse un prejuicio implícito en general en favor de los negros y en contra de los blancos (esto sería como idealismo de superioridad africana).

Tabla N..$^{\circ} 2$

Diferencias de Media, por bloques

\begin{tabular}{|c|c|c|c|c|c|c|c|c|}
\hline & \multicolumn{5}{|c|}{ Diferencias emparejadas } & \multirow{3}{*}{$\mathrm{t}$} & \multirow{3}{*}{ Gl } & \multirow{3}{*}{$\begin{array}{c}\text { Sig. } \\
\text { (bilateral) }\end{array}$} \\
\hline & \multirow[t]{2}{*}{ Media } & \multirow[t]{2}{*}{$\begin{array}{l}\text { Desviación } \\
\text { estándar }\end{array}$} & \multirow[t]{2}{*}{$\begin{array}{l}\text { Media } \\
\text { de error } \\
\text { estándar }\end{array}$} & \multicolumn{2}{|c|}{$\begin{array}{c}95 \% \text { de intervalo } \\
\text { de confianza de la } \\
\text { diferencia }\end{array}$} & & & \\
\hline & & & & Inferior & Superior & & & \\
\hline $\begin{array}{l}\text { Par 1 } \\
\text { latency_blo- } \\
\text { que_6_ms - } \\
\text { latency_blo- } \\
\text { que_3_ms }\end{array}$ & -4939.13 & 13509.77 & 1259.79 & -7434.77 & -2443.49 & -3.92 & 114 & .000 \\
\hline $\begin{array}{c}\text { Par } 2 \\
\text { latency_blo- } \\
\text { que_7_ms - } \\
\text { latency_blo- } \\
\text { que_4_ms }\end{array}$ & -895.65 & 9411.06 & 877.59 & -2634.14 & 842.84 & -1.02 & 114 & .310 \\
\hline
\end{tabular}

\begin{tabular}{|c|c|c|c|c|c|c|c|c|}
\hline & \multicolumn{5}{|c|}{ Diferencias emparejadas } & \multirow{3}{*}{$\mathrm{t}$} & \multirow{3}{*}{ Gl } & \multirow{3}{*}{$\begin{array}{c}\text { Sig. } \\
\text { (bilateral) }\end{array}$} \\
\hline & \multirow[t]{2}{*}{ Media } & \multirow[t]{2}{*}{$\begin{array}{c}\text { Desviación } \\
\text { estándar }\end{array}$} & \multirow[t]{2}{*}{$\begin{array}{l}\text { Media } \\
\text { de error } \\
\text { estándar }\end{array}$} & \multicolumn{2}{|c|}{$\begin{array}{c}95 \% \text { de intervalo } \\
\text { de confianza de la } \\
\text { diferencia }\end{array}$} & & & \\
\hline & & & & \begin{tabular}{|l|} 
Inferior \\
\end{tabular} & Superior & & & \\
\hline $\begin{array}{c}\text { Par1 } \\
\text { dif_B6_B3 } \\
\text { dif_B7_B4 } \\
\end{array}$ & -4043.48 & 14642.16 & 1365.39 & -6748.30 & -1338.65 & -2.96 & 114 & .004 \\
\hline
\end{tabular}


No existen diferencias significativas en los resultados por variables demográficas (edad, sexo y raza), es una posibilidad que el tamaño reducido de la muestra (115 casos), así como la metodología de selección muestral influya en estos resultados.

Tabla N. ${ }^{\circ} 3$

Pruebas de efectos inter-sujetos

\begin{tabular}{|l|r|r|r|r|r|}
\hline \multicolumn{1}{|c|}{ Origen } & $\begin{array}{c}\text { Tipo III de } \\
\text { suma de } \\
\text { cuadrados }\end{array}$ & g1 & $\begin{array}{r}\text { Cuadrático } \\
\text { promedio }\end{array}$ & F & Sig. \\
\hline Modelo corregido & $42.756^{a}$ & 35 & 1.222 & 1.342 & .142 \\
Interceptación & .340 & 1 & .340 & .374 & .543 \\
Sexo & 4.991 & 1 & 4.991 & 5.485 & .022 \\
P2 & 4.682 & 5 & .936 & 1.029 & .407 \\
Edad_Transformada & 6.086 & 4 & 1.522 & 1.672 & .165 \\
E1 * P2 & 4.015 & 4 & 1.004 & 1.103 & .361 \\
E1 * Edad_Transformada & 2.626 & 3 & .875 & .962 & .415 \\
P2 $*$ Edad_Transformada & 14.745 & 10 & 1.475 & 1.620 & .116 \\
E1 $*$ P2 $*$ Edad_Transformada & 6.487 & 8 & .811 & .891 & .528 \\
Error & 70.978 & 78 & .910 & & \\
Total & 113.736 & 114 & & & \\
Total corregido & 113.734 & 113 & & & \\
\hline
\end{tabular}

Variable dependiente: Z_dif_B6_B3

a. $\mathrm{R}$ al cuadrado $=.376(\mathrm{R}$ al cuadrado ajustada $=.096)$

Aunque se observa significancia en la variable sexo, las pruebas de contraste más profundas descartan esta variable, para ajustarse a un modelo. La varianza en la variable sexo es igual a 0 ; dicho de otro modo, no existe diferencia entre los hombres y las mujeres en la velocidad de selección de los bloques (Sig. > 0.05) (Ver estadístico de Levene, en la tabla N. ${ }^{\circ}$ ). 
Tabla N. ${ }^{\circ} 4$

ANOVA Sexo * Diferencia de Bloques

ANOVA

\begin{tabular}{|l|r|c|r|r|l|}
\hline & $\begin{array}{r}\text { Suma de } \\
\text { cuadrados }\end{array}$ & gl & $\begin{array}{c}\text { Media } \\
\text { cuadrática }\end{array}$ & F & Sig. \\
\hline Z_dif_B6_B3 & & & & & \\
Entre grupos & 1.333 & 1 & 1.333 & 1.338 & .250 \\
Dentro de grupos & 112.540 & 113 & .996 & & \\
Total & 113.872 & 114 & & & \\
\hline Z_dif_B7_B4 & & & & & \\
Entre grupos & 1.284 & 1 & 1.284 & 1.287 & .259 \\
Dentro de grupos & 112.716 & 113 & .997 & & \\
Total & 114.000 & 114 & & & \\
\hline
\end{tabular}

Prueba de homogeneidad de varianzas

\begin{tabular}{|l|r|c|c|c|}
\hline & $\begin{array}{c}\text { Estadístico } \\
\text { de Levene }\end{array}$ & df1 & df2 & Sig. \\
\hline Z_dif_B6_B3 & 1.064 & 1 & 113 & .305 \\
Z_dif_B7_B4 & .566 & 1 & 113 & .454 \\
\hline
\end{tabular}

\subsection{Estadísticas y modelo de actitud explícita}

Para la medición explícita fueron utilizadas variables de autopercepción de la raza y el color de piel, así como el juicio de un evaluador sobre estas variables. A la vez fueron motivados a responder a través de una escala de Likert una serie de afirmaciones sobre dominio y prejuicio racial; otro componente de la aplicación fue un termómetro racial sobre la comodidad de interactuar con personas de diferente raza. 
Los entrevistados perciben su color de piel más claro de cómo es observado por otras personas, en este caso el puntaje medio del color de piel de los entrevistados es inferior al observado por el entrevistador, siendo este valor significativo a un nivel de confianza de $94 \%$. Esto es un hallazgo que es consistente con la literatura, ya que los autores Sidanius, Peña, \& Sawyer (2001) han encontrado lo mismo al realizar su estudio de percepción racial en el público en general de la República Dominicana.

\section{Tabla N. ${ }^{\circ} 5$}

Análisis de medias

(Comparación entre la percepción de tono de piel del participante y la observación de evaluador)

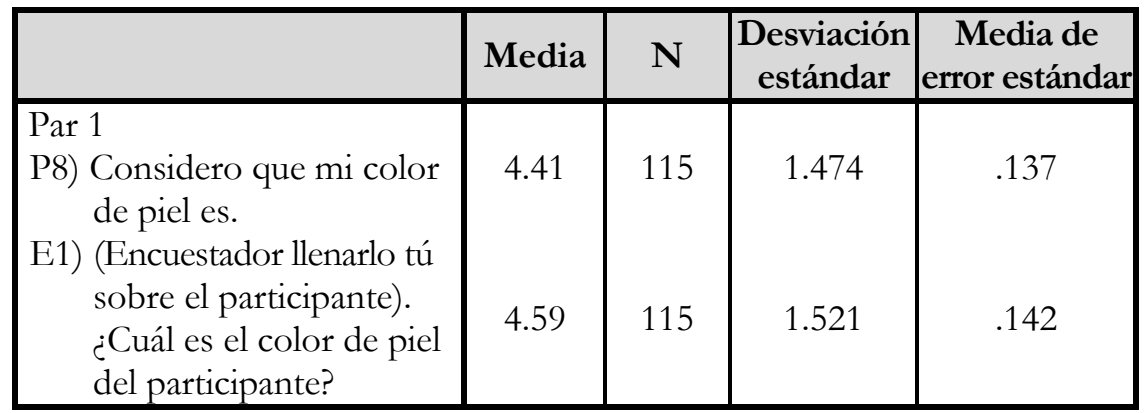

\begin{tabular}{|c|c|c|c|c|c|c|c|c|}
\hline & \multicolumn{5}{|c|}{ Diferencias emparejadas } & \multirow[b]{2}{*}{$t$} & \multirow[b]{2}{*}{$\mathrm{gl}$} & \multirow[b]{2}{*}{$\begin{array}{c}\text { Sig. } \\
\text { (bilateral }\end{array}$} \\
\hline & Media & $\begin{array}{c}\text { Desviación } \\
\text { estándar }\end{array}$ & $\begin{array}{l}\text { Media de } \\
\text { error } \\
\text { estándar }\end{array}$ & \begin{tabular}{|r}
$\begin{array}{r}95 \% \text { de } \\
\text { de conf } \\
\text { la dife }\end{array}$ \\
Inferior \\
\end{tabular} & $\begin{array}{l}\text { intervalo } \\
\text { fianza de } \\
\text { ferencia } \\
\text { Superior }\end{array}$ & & & \\
\hline $\begin{array}{l}\text { Par } 1 \\
\text { P8) Considero } \\
\text { que mi color } \\
\text { de piel es. } \\
\text { E1) (Encuestador } \\
\text { llenarlo tu so- } \\
\text { bre el partici- } \\
\text { pante) ¿Cuál } \\
\text { es el color de } \\
\text { piel del parti- } \\
\text { cipante? }\end{array}$ & -.183 & 1.039 & .097 & -.375 & .009 & -1.89 & 114 & .062 \\
\hline
\end{tabular}


La actitud explícita fue calculada a través de tres variables con afirmaciones sobre percepción racial, las cuales fueron respondidas a través de una escala de Likert. Estas a su vez fueron consolidadas en una dimensión, utilizando la técnica de análisis factorial, la cual es capaz de reducir un conjunto de variables en una cantidad eficiente de dimensiones, con la menor pérdida de información posible. Las pruebas estadísticas para realizar este procedimiento son positivas y recomendadas para reducir las variables (ver tabla N. ${ }^{\circ} 8$ ), observándose que la adecuación del muestreo del estadístico KMO es aceptable > .664; así como la prueba de esfericidad de Bartlet indica que la matriz no es de identidad (>.000); por lo cual se procedió con el procedimiento. El factor resultante representa el $71.3 \%$ de la varianza (véase la tabla N. ${ }^{\circ} 9$ ).

Tabla N. ${ }^{\circ} 6$

Afirmaciones y puntajes de componentes principales

\begin{tabular}{|l|c|}
\hline \multirow{2}{*}{} & Componente \\
\cline { 2 - 2 } & 1 \\
\hline P6) ¿Cuál de las siguientes afirmaciones & .765 \\
te describe mejor? & .869 \\
P7) ¿Personas de piel oscura? & .893 \\
P7) \{Personas de piel clara\} & \\
\hline
\end{tabular}

Método de extracción: análisis de componentes principales.

a. 1 componentes extraídos.

\section{Tabla N. ${ }^{\circ} 7$}

\section{Prueba de KMO y Bartlett}

\begin{tabular}{|c|c|c|}
\hline \multicolumn{2}{|c|}{$\begin{array}{l}\text { Medida Kaiser-Meyer-Olkin de adecuación de } \\
\text { muestreo }\end{array}$} & .664 \\
\hline \multirow{3}{*}{$\begin{array}{c}\text { Prueba de esfericidad } \\
\text { de Bartlett }\end{array}$} & Aprox. Chi-cuadrado & 116.839 \\
\hline & Gl & 3 \\
\hline & Sig. & .000 \\
\hline
\end{tabular}


Tabla N. ${ }^{\circ} 8$

Varianza total explicada

\begin{tabular}{|c|c|c|c|c|c|c|}
\hline \multirow{2}{*}{ Componente } & \multicolumn{2}{|c|}{ Autovalores iniciales } & \multicolumn{3}{c|}{$\begin{array}{c}\text { Sumas de extracción } \\
\text { de cargas al cuadrado }\end{array}$} \\
\cline { 2 - 6 } & Total & $\begin{array}{c}\% \text { de } \\
\text { varianza }\end{array}$ & $\begin{array}{c}\% \\
\text { acumulado }\end{array}$ & Total & $\begin{array}{c}\% \text { de } \\
\text { varianza }\end{array}$ & $\begin{array}{c}\% \\
\text { acumulado }\end{array}$ \\
\hline 1 & 2.138 & 71.262 & 71.262 & 2.138 & 71.262 & 71.262 \\
2 & .575 & 19.173 & 90.434 & & & \\
3 & .287 & 9.566 & 100.000 & & & \\
\hline
\end{tabular}

Método de extracción: análisis de componentes principales.

El factor resultante con el procedimiento del Análisis Factorial, por componentes principales y rotación Varimax ha sido denominado Termómetro Racial2; sus valores fueron acotados y escalados en valores comprendidos desde el 1 al 7 . El termómetro es interpretado como: "a mayor valor, más preferencia existe peor personas de piel oscura; mientras que a menor valor mayor preferencia por personas de piel clara.

\subsection{Termómetro por variables socioeconómicas}

Los datos del termómetro racial confirman los hallazgos de la actitud implícita, sobre la preferencia de los participantes hacia personas con piel de color oscura (4.07); observándose que en las mujeres (sig. $<0.05$ ). La preferencia hacia este color de piel es menor.

2 El modelo para el Termómetro $=0.765$ P3 +0.869 P7.a +0.83 P7.b) 
Tabla N. ${ }^{\circ} 9$

Medias del termómetro * Género

\begin{tabular}{|l|c|c|c|}
\hline Género & Media & N & $\begin{array}{c}\text { Desviación } \\
\text { estándar }\end{array}$ \\
\hline Femenino & 3.69 & 59 & 2.062 \\
Masculino & 4.46 & 56 & 1.926 \\
Total & 4.07 & 115 & 2.025 \\
\hline
\end{tabular}

Es importante puntualizar que los individuos con menor edad presentan mayor preferencia (sig. $<0.00$ ) hacia personas con tonos de piel más claros; incrementándose la tendencia a la preferencia de personas con piel de color más oscura mientras se va envejeciendo. No se identificaron diferencias significativas en el termómetro por raza, nivel académico y trimestre.

Tabla N. ${ }^{\circ} 10$

Medias del termómetro $*$ Edad

\begin{tabular}{|c|c|c|c|}
\hline $\begin{array}{c}\text { P1) } \\
\text { Edad: (Binned) }\end{array}$ & Media & $\mathbf{N}$ & $\begin{array}{c}\text { Desviación } \\
\text { estándar }\end{array}$ \\
\hline$<=17$ & 2.00 & 2 & 1.414 \\
$18-20$ & 3.22 & 51 & 2.110 \\
$21-23$ & 4.62 & 40 & 1.612 \\
$24-26$ & 5.24 & 17 & 1.640 \\
$27-28$ & 5.50 & 4 & 1.732 \\
Total & 4.07 & 114 & 2.034 \\
\hline
\end{tabular}

\section{DOMINANCIA Y PREJUICIO RACIAL}

La dominancia y el prejuicio racial han sido calculados por medio de una batería de preguntas con 6 afirmaciones medidas en una escala de Likert (Muy en desacuerdo - Muy de acuerdo). 
Tabla N. ${ }^{\circ} 11$

Estadísticos descriptivos

\begin{tabular}{|c|c|c|c|c|c|}
\hline & N & Mínimo & Máximo & Media & $\begin{array}{c}\text { Desviación } \\
\text { estándar }\end{array}$ \\
\hline $\begin{array}{c}\text { P10) Para progresar en la vida, a veces } \\
\text { es necesario pisar a los demás. }\end{array}$ & 115 & 1 & 6 & 2.64 & 1.133 \\
$\begin{array}{c}\text { P11) Probablemente es bueno que } \\
\text { algunos grupos estén en la cima } \\
\text { y otros en el fondo. }\end{array}$ & 115 & 1 & 6 & 3.10 & 1.202 \\
$\begin{array}{c}\text { P12) Si algunos grupos se quedasen } \\
\text { en su lugar, tendríamos menos } \\
\text { problemas. }\end{array}$ & 115 & 1 & 6 & 2.83 & 1.147 \\
$\begin{array}{c}\text { P13) Debemos luchar para que todos } \\
\text { tengamos un salario equitativo. }\end{array}$ & 115 & 1 & 6 & 3.74 & 1.639 \\
$\begin{array}{c}\text { P14) Las costumbres tradicionales y } \\
\text { los valores tradicionales son la } \\
\text { mejor manera de vivir. }\end{array}$ & 115 & 1 & 6 & 3.92 & 1.427 \\
$\begin{array}{c}\text { P15) Nuestro país necesita un líder } \\
\text { fuerte para poder terminar con } \\
\text { los grupos radicales e inmorales. }\end{array}$ & 115 & 1 & 6 & 3.47 & 1.552 \\
N válido (por lista)
\end{tabular}

Fue utilizado el análisis factorial con componentes principales y rotación Varimax para reducir estas variables en dos dimensiones, las cuales representan facilidad de interpretación y análisis. Las pruebas (ver tabla para proceder con esta técnica fueron positivas, resultando conveniente realizarlo. La adecuación del muestreo de $\mathrm{KMO}$ es aceptable > 0.5; así como prueba de esfericidad de Bartlet indica que la matriz no es de identidad (>.000); por lo cual se procedió con el procedimiento. Los factores resultantes explican el $54.4 \%$ de la varianza. 
Tabla N. ${ }^{\circ} 12$

Prueba de KMO y Bartlett

\begin{tabular}{|c|c|c|}
\hline \multicolumn{2}{|l|}{$\begin{array}{l}\text { Medida Kaiser-Meyer-Olkin de adecuación de } \\
\text { muestreo }\end{array}$} & .607 \\
\hline \multirow{2}{*}{$\begin{array}{c}\text { Prueba de esfericidad } \\
\text { de Bartlett }\end{array}$} & Aprox. Chi-cuadrado & 81.739 \\
\cline { 2 - 3 } & Gl & 15 \\
\cline { 2 - 3 } & Sig. & .000 \\
\hline
\end{tabular}

Tabla N. ${ }^{\circ} 13$

Varianza total explicada

\begin{tabular}{|c|c|c|c|c|c|c|c|c|c|}
\hline \multirow{2}{*}{ Comp. } & \multicolumn{3}{|c|}{ Autovalores iniciales } & \multicolumn{3}{|c|}{$\begin{array}{l}\text { Sumas de extracción } \\
\text { de cargas al cuadrado }\end{array}$} & \multicolumn{3}{|c|}{$\begin{array}{c}\text { Sumas de rotación } \\
\text { de cargas al cuadrado }\end{array}$} \\
\hline & Total & $\begin{array}{c}\% \text { de } \\
\text { varianza }\end{array}$ & $\begin{array}{c}\% \\
\text { acumulado }\end{array}$ & Total & \begin{tabular}{|c|}
$\%$ de \\
varianza
\end{tabular} & $\begin{array}{c}\% \% \\
\text { acumulado }\end{array}$ & Total & $\begin{array}{c}\% \text { de } \\
\text { varianza }\end{array}$ & $\begin{array}{c}\% \% \\
\text { acumulado }\end{array}$ \\
\hline 1 & 1.987 & 33.117 & 33.117 & 1.987 & 33.117 & 33.117 & 1.667 & 27.790 & 27.790 \\
\hline 2 & 1.279 & 21.321 & 54.438 & 1.279 & 21.321 & 54.438 & 1.599 & 26.648 & 54.438 \\
\hline 3 & .931 & 15.510 & 69.949 & & & & & & \\
\hline 4 & .781 & 13.025 & 82.974 & & & & & & \\
\hline 5 & .551 & 9.181 & 92.155 & & & & & & \\
\hline 6 & .471 & 7.845 & 100.000 & & & & & & \\
\hline
\end{tabular}

Método de extracción: análisis de componentes principales.

Las dos dimensiones extraídas (ver tabla N. ${ }^{\circ}$ 15) fueron denominadas: Dominancia ${ }^{3}$ y Cohesión ${ }^{4}$; para facilitar el análisis e interpretación de los puntajes de estas dimensiones fueron acotados y escalados con valores del 1 al 6. Interpretándose como: a mayor valor en la dimensión mayor es el nivel expresado de Dominancia y/o Cohesión.

\footnotetext{
${ }^{3}$ Dominancia $=0.64 \mathrm{P} 10+0.700 \mathrm{P} 11+0.541 \mathrm{P} 12-0.690 \mathrm{P} 13-0.461 \mathrm{P} 14-0.320 \mathrm{P} 15$

${ }^{4}$ Cohesion $=-0.12 \mathrm{P} 10+0.481 \mathrm{P} 11+0.599 \mathrm{P} 12+0.339 \mathrm{P} 13+0.682 \mathrm{P} 14+0.330 \mathrm{P} 15$
}

100 Ciencia y Sociedad 2016; 41(1): 77-111 


\section{Tabla N. ${ }^{\circ} 14$}

Matriz de componente rotado

\begin{tabular}{|c|c|c|}
\hline & \multicolumn{2}{|c|}{ Componente } \\
\hline & $\begin{array}{c}1 \\
\text { Dominancia }\end{array}$ & $\begin{array}{c}2 \\
\text { Cohesión }\end{array}$ \\
\hline $\begin{array}{l}\text { P10) Para progresar en la vida, a veces es necesario } \\
\text { pisar a los demás }\end{array}$ & .468 & \\
\hline $\begin{array}{l}\text { P11) Probablemente es bueno que algunos grupos } \\
\text { estén en la cima y otros en el fondo. }\end{array}$ & .842 & \\
\hline $\begin{array}{l}\text { P12) Si algunos grupos se quedasen en su lugar, } \\
\text { tendríamos menos problemas. }\end{array}$ & .803 & \\
\hline $\begin{array}{l}\text { P13) Debemos luchar para que todos tengamos } \\
\text { un salario equitativo. }\end{array}$ & & .714 \\
\hline $\begin{array}{l}\text { P14) Las costumbres tradicionales y los valores } \\
\text { tradicionales son la mejor manera de vivir. }\end{array}$ & & .815 \\
\hline $\begin{array}{l}\text { P15) Nuestro país necesita un líder fuerte para } \\
\text { poder terminar con los grupos radicales e } \\
\text { inmorales. }\end{array}$ & & .459 \\
\hline
\end{tabular}

Método de extracción: análisis de componentes principales.

Método de rotación: Varimax con normalización Kaiser.

a. La rotación ha convergido en 3 iteraciones

\section{DOMINANCIA Y COHESIÓN POR NIVELES SOCIO DEMOGRÁFICOS}

En total, tanto las dimensiones de dominancia como la cohesión presentan valores medios cercanos a 3.5 en la escala (1-6). Existe una diferencia estadísticamente significativa $(<0.05)$ entre la percepción de dominancia y cohesión; observándose que las mujeres tienen mayor tendencia a la dimensión de cohesión que los hombres los cuales tienen una tendencia marcada a la dominancia. Aunque no presentan diferencias estadísticamente significativas, es importante observar que los grupos con colores más oscuros tienden a tener valores mayores en la dimensión de dominancia que las razas más claras, esto se acerca bastante a los resultados de la actitud implícita, sobre la dominancia racial africana. 
Gráfico $\mathbf{N}^{\circ}{ }^{1}$

Promedio de niveles de dominancia racial y cohesion por género

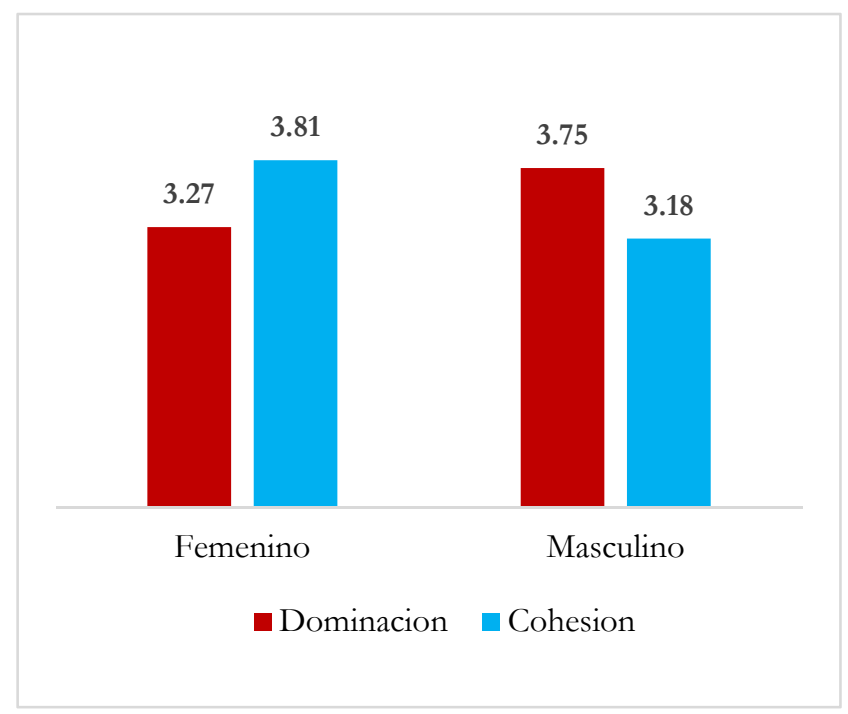

Gráfico $\mathrm{N}^{\circ}{ }^{\circ}$

Raza por dimensión de dominancia

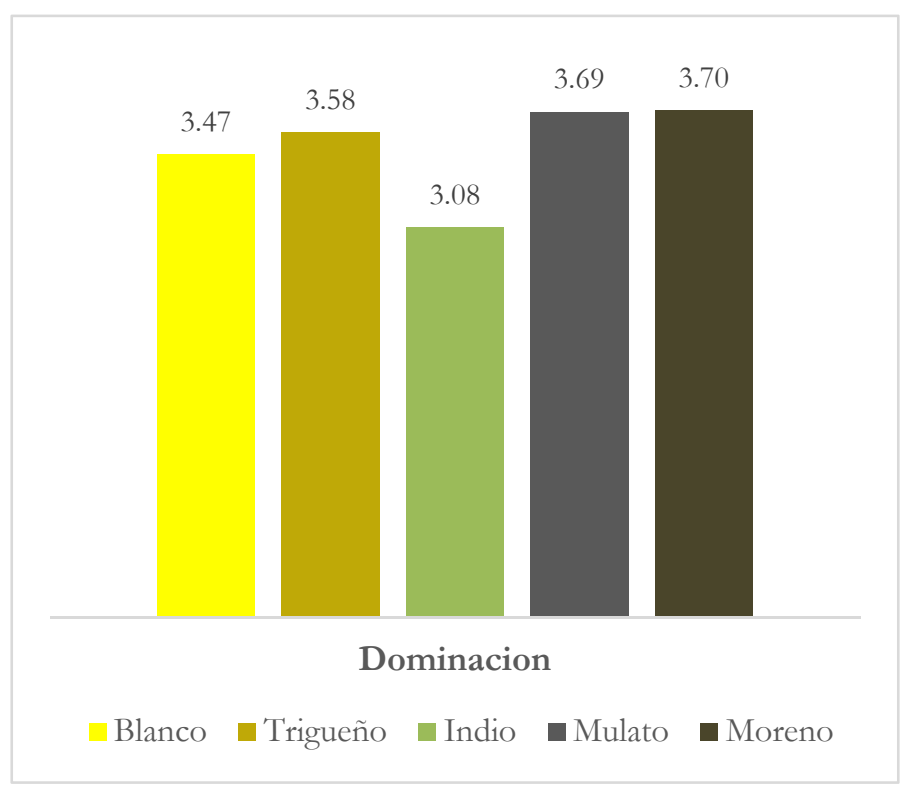

102 Ciencia y Sociedad 2016; 41(1): 77-111 
La edad es una variable de importancia sobre la Dominancia (ver tabla N. ${ }^{\circ}$ 16), mientras más se incrementa la edad, el nivel de dominancia también se incrementa; ocurriendo el efecto inverso en la relación de la edad con la dimensión Cohesión.

Tabla N. ${ }^{\circ} 15$

Medias de edad * dimensiones dominación y cohesión

\begin{tabular}{|c|c|c|}
\hline P1) Edad: (Binned) & Dominación & Cohesión \\
\hline$<=17$ & 2.00 & 5.50 \\
$18-20$ & 3.06 & 4.14 \\
$21-23$ & 3.72 & 3.00 \\
$24-26$ & 4.24 & 2.53 \\
$27-28$ & 4.50 & 3.25 \\
Total & 3.50 & 3.49 \\
\hline
\end{tabular}

Tabla N. ${ }^{\circ} 17$

Medidas de asociación

\begin{tabular}{|l|c|c|c|c|}
\hline & $\mathbf{R}$ & $\begin{array}{c}\mathbf{R} \text { al } \\
\text { cuadrado }\end{array}$ & Eta & $\begin{array}{c}\text { Eta } \\
\text { cuadrada }\end{array}$ \\
\hline Dominación * P1) Edad: (Binned) & .293 & .086 & .299 & .089 \\
Cohesión * P1) Edad: (Binned) & -.355 & .126 & .408 & .166 \\
\hline
\end{tabular}


Tabla N. ${ }^{\circ} 16$

Tabla de ANOVA

\begin{tabular}{|c|c|c|c|c|c|c|c|}
\hline & & & $\begin{array}{l}\text { Suma de } \\
\text { cuadrados }\end{array}$ & gl & $\begin{array}{c}\text { Media } \\
\text { cuadrática }\end{array}$ & $\mathbf{F}$ & Sig. \\
\hline \multirow{5}{*}{$\begin{array}{l}\text { Dominación } \\
\text { * P1) Edad: } \\
\text { (Binned) }\end{array}$} & \multirow{3}{*}{$\begin{array}{l}\text { Entre } \\
\text { grupos }\end{array}$} & Combinado & 29.643 & 4 & 7.411 & 2.667 & .036 \\
\hline & & Linealidad & 28.548 & 1 & 28.548 & 10.274 & .002 \\
\hline & & $\begin{array}{l}\text { Desviación de } \\
\text { la linealidad }\end{array}$ & 1.095 & 3 & .365 & .131 & .941 \\
\hline & \multicolumn{2}{|c|}{ Dentro de grupos } & 302.857 & 109 & 2.779 & & \\
\hline & \multicolumn{2}{|l|}{ Total } & 332.500 & 113 & & & \\
\hline \multirow{5}{*}{$\begin{array}{l}\text { Cohesión } \\
\text { * P1) Edad: } \\
\text { (Binned) }\end{array}$} & \multirow{3}{*}{$\begin{array}{l}\text { Entre } \\
\text { grupos }\end{array}$} & Combinado & 54.967 & 4 & 13.742 & 5.436 & .000 \\
\hline & & Linealidad & 41.759 & 1 & 41.759 & 16.520 & .000 \\
\hline & & $\begin{array}{l}\text { Desviación de } \\
\text { la linealidad }\end{array}$ & 13.208 & 3 & 4.403 & 1.742 & .163 \\
\hline & \multicolumn{2}{|c|}{ Dentro de grupos } & 275.525 & 109 & 2.528 & & \\
\hline & \multicolumn{2}{|l|}{ Total } & 330.491 & 113 & & & \\
\hline
\end{tabular}

\section{Conclusiones y recomendaciones}

Con los hallazgos analizados en el grupo objeto de estudio puede inferirse que en la República Dominicana no existe la denominada democracia racial propuesta por freyre en el 1951. En este caso la evidencia nos aproxima a la tesis de dominancia social planteada por Sidanius en 1993.

Tanto a nivel de subconsciente (actitud implícita) como consciente reportado (actitud explícita) los participantes en el estudio presentan prejuicio implícito en general en favor de los negros y en contra de los blancos, es decir, idealismo de superioridad africana, de forma explícita las mujeres presentan un menor idealismo de superioridad africana que los hombres. Puede ser que la población bajo estudio tenga mayor conciencia de la descendencia racial y se sienta más cómodo con su herencia africana o pudiera ser que al ser mayoría, los de piel oscura se perciben como que deberían dominar.

104 Ciencia y Sociedad 2016; 41(1): 77-111 
Los individuos con menor edad presentan mayor preferencia hacia personas con tonos de piel más claros; incrementándose la tendencia a la preferencia de personas con piel de color más oscura mientras se va envejeciendo. No se identificaron diferencias significativas en el Termómetro por raza, nivel académico y ni por trimestre.

Un fenómeno que fue observado en el estudio es que las personas perciben su color de piel más claro de cómo es observado por otras personas, aunque cuando especifican su raza lo la identifican con un tono más oscuro de la que realmente tiene.

En cuanto a la dominancia racial, las mujeres tienen mayor tendencia hacia la cohesión social respecto a los hombres, los cuales tienen una preferencia marcada a la dominancia. Aunque no fueron evidenciadas diferencias estadísticamente significativas es importante observar que los grupos con colores más oscuros tienden a tener valores mayores y mayor niveles de dominancia con relación a las razas más claras, esto se acerca bastante a los resultados de la actitud implícita, sobre la dominancia racial africana.

La edad es una variable de importancia sobre la dominancia, mientras más se incrementa la edad, el nivel de dominancia también se incrementa; ocurriendo el efecto inverso en la relación de la edad con la dimensión cohesión.

\subsection{Recomendaciones}

El alcance y la representatividad muestral de este estudio debería ser incrementado a futuro, esto con la finalidad de profundizar de forma precisa sobre este fenómeno en toda la población de República Dominicana, la cual en este caso por limitaciones de tiempo y recursos fue realizada a una muestra que no garantiza la representación a todos individuos o grupos de dominicanos. 


\section{Referencias}

Banse, R., Seise, J. \& Zerbes, N. (2001). Implicit attitudes towards homosexuality: Reliability, validity, and control- lability of the IAT. Zeitschrift fuer Experimentelle Psychologie, 48(2), 145-160.

Bargh, J. A., Chen, M. \& Burrows, L. (1996). Automaticity of social behavior: Direct effects of trait construct and stereotype activation on action. Journal of Personality and Social Psychology, 71(2), 230-244.

Blair, I. V. (2001). Implicit stereotypes and prejudice. In G. B. Moskowitz (Ed.). Cognitive social psychology: The Princeton Symposium on the Legacy and Future of Social Cognition (pp. 359-374). Mahwah, NJ: Lawrence Erlbaum.

Bosson, J. K., Swann, W. B. \& Pennebaker, J. W. (2000). Stalking the perfect measure of implicit self-esteem: The blind men and the elephant revisited? Journal of Personality and Social Psychology, 79(4), 631-643.

Carver, C. S., Glass, D. C. \& Katz, I. (1978). Favorable evaluations of Blacks and the handicapped: Positive prejudice, unconscious denial, or social desirability? Journal of Applied Social Psychology, 8, 97-106.

Central Intelligence Agency. (2000). World factbook 2001. Recuperado de http://www.odci.gov/cia/publications/factbook /index.html

Chen, M. \& Bargh, J. A. (1997). Nonconscious behavioral confirmation processes: The self-fulfilling consequences of automatic stereotype activation. Journal of Experimental Social Psychology, 33(5), 541-560. 
Correll, J., Park, B., Judd, C. M. \& Wittenbrink, B. (2002). The police officer's dilemma: Using ethnicity to disambiguate potentially threatening individuals. Journal of Personality and Social Psychology, 83, 1314-1329.

Crosby, F., Bromley, S. \& Saxe, L. (1980). Recent unobtrusive studies of Black and White discrimination and prejudice: A literature review. Psychological Bulletin, 87(3), 546-563.

Degler, C. (1986). Neither Black nor White: Slavery and race relations in the United States and Brazil. Madison: University of Wisconsin Press.

Dijksterhuis, A., \& Bargh, J. A. (2001). The perception-behavior expressway: Automatic effects of social perception on social behavior. In M. P. Zanna (Ed.). Advances in experimental social psychology (Vol. 33, pp. 1-40). San Diego: Academic Press.

Dovidio, J. F. \& Fazio, R. H. (1992). New technologies for the direct and indirect assessment of attitudes. In J. M. Tanur (Ed.). Questions about questions: Inquiries into the cognitive bases of surveys (pp. 204-237). New York: Russell Sage Foundation.

Duany, J. (2001). Making Indians out of Blacks: The revitalization of Taíno identity in contemporary Puerto Rico. In G. Haslip-Viera (Ed.). Taino revival: Critical perspectives on Puerto Rican identity and cultural politics (pp. 55-82). New Jersey: Markus Wiener.

Fazio, R. H., Jackson, J. R., Dunton, B. C. \& Williams, C. J. (1995). Variability in automatic activation as a unob- trusive measure of racial attitudes: A bona fide pipeline? Journal of Personality and Social Psychology, 69, 1013-1027.

Freyre, G. (1951). Brazil: An interpretation. New York: Alfred Knopf. 
Fuente, A., de la. (1995). Race and inequality in Cuba, 1899-1981. Journal of Contemporary History, 30, 131-168.

Gaertner, S. L. \& McLaughlin, J. P. (1983). Racial stereotypes: Associations and ascriptions of positive and negative characteristics. Social Psycbology Quarterly, 46, 23-30.

Greenwald, A. G., McGhee, D. E. \& Schwartz, J. L. (1998). Measuring individual differences in implicit cognition: The implicit association test. Journal of Personality and Social Psychology, 74(6), 1464-1480.

Greenwald, A. G. \& Nosek, B. A. (2001). Health of the Implicit Association Test at age 3. Zeitschrift fuer Experimentelle Psychologie, 48(2), 85-93.

Guimaraes, A. S. (2002). Democracia racial: el dieal, el pacto y el mito. Revista Estudios Sociológicos, 49 (59) 305-333.

Hanchard, M. G. (1994). Orpheus and power: The movement negro of Rio de Janeiro and São Paulo, Brazil, 1945-1988. New Jersey: Princeton University Press.

Hoetink, H. (1967). Caribbean race relations. London: Oxford University Press.

Humboldt, A. (1990). Historia de la esclavitud en la isla [History of slavery on the island]. In Textos de la historia de Centroamérica y el caribe: Cuba 1 (pp. 335-338). Guadalajara, México: Nueva Imagen.

Jost, J. T., Burgess, D. \& Mosso, C. O. (2001). Conflicts of legitimization among self, group, and system: The integrative potential of system justification theory. In J. T. Jost \& B. Major (Eds.). The psychology of legitimacy: Emerging perspectives on ideology, justice, and intergroup relations (pp. 363-391). New York: Cambridge University Press. 
Kuehnen, U., Schiessl, M., Bauer, N., Paulig, N., Poehlmann, C. \& Schmidthals, K. (2001). How robust is the IAT? Measuring and manipulating implicit attitudes of East- and West-Germans. Zeitschrift fuer Experimentelle Psychologie, 48(2), 135-144.

Lee, T. (2002). Mobilizing Black public opinion: Black insurgency and racial attitudes in the civil rights era. Chicago: University of Chicago Press.

Marx, A. W. (1998). Making race and nation: A comparison of the United States, South Africa and Brazil. New York: Cambridge University Press.

McConnell, A. R. \& Leibold, J. M. (2001). Relations among the Implicit Association Test, discriminatory behavior, and explicit measures of racial attitudes. Journal of Experimental Social Psychology, 37, 435-442.

Minority Rights Group. (1995). No longer invisible. London: Minority Rights Publications.

Moya-Pons, F. (2000). Manual de historia dominicana [Dominican historical manual. Santo Domingo: Caribbean Publishers.

Nobles, M. (2000). Shades of citizenship: Race and the census in modern politics. Stanford: Stanford University Press.

Payne, B. K. (2001). Prejudice and perception: The role of automatic and controlled processes in misperceiving a weapon. Journal of Personality and Social Psychology, 81, 181-192.

Pierson, D. (1942). Negroes in Brazil: A study of race contact at Bahia. Chicago: University of Chicago Press. Ridgeway, C. L. (2001). The emergence of status beliefs: From structural inequality to legitimizing ideology. In J. T. Jost \& B. Major (Eds.). The psychology of legitimacy: Emerging perspectives on ideology, justice, and inter- group relations (pp. 257-306). New York: Cambridge University Press. 
Sidanius, J., Levin, S., Federico, C. \& Pratto, F. (2001). Legitimizing ideologies: The social dominance approach. In J. T. Jost \& B. Major (Eds.). The psychology of legitimacy: Emerging perspectives on ideology, justice, and inter- group relations (pp. 307-331). New York: Cambridge University Press.

Sidanius, J., Peña, Y. \& Sawyer, M. (2001). Inclusionary Discrimination: Pigmentocracy and Patriotism in the Dominican Republic. Political Psychology, 22(4), 827-851.

Twine, F. W. (1998). Racism in a racial democracy: The maintenance of White supremacy. New Brunswick. New Jersey: Rutgers University Press.

Wade, P. (1997). Race and ethnicity in Latin America. London: Pluto Press.

Wittenbrink, B., Judd, C. M. \& Park, B. (1997). Evidence for racial prejudice at the implicit level and its relationship with questionnaire measures. Journal of Personality and Social Psychology, 72(2), 262-274.

Wittenbrink, B., Judd, C. M. \& Park, B. (2001). Spontaneous prejudice in context: Variability in automatically activated attitudes. Journal of Personality and Social Psychology, 81, 815-827. 


\section{Yesilernis L. Peña}

Posee un Ph.D. en Psicología Social-Industrial con una concentración en Metodología/Estadística de la Universidad de California, Los Ángeles (UCLA) y cuenta con más de 15 años de experiencia en investigaciones. Ha realizado un sinnúmero de investigaciones tanto en los Estados Unidos como en Latinoamérica (México, Venezuela, Brasil, Puerto Rico, Cuba y República Dominicana) que han sido publicadas en varias revistas científicas internacionales y sus respectivas presentaciones en congresos internacionales. Ha desarrollado proyectos de investigación auspiciados por instituciones internacionales, como National Science Foundation (NSF), Ford Foundation, American Psicological Asociation (APA); y a nivel nacional, ha recibido apoyo del IDEICE, MINERD, MITUR, PNUD, Ministerio de Educación Superior de Ciencia y Tecnología (MESCyT), incluyendo FONDOCy'T, entre tanto otras. De igual modo, la dra. Peña ha colaborado con diversas universidades internacionales en proyectos de investigación, como con UCLA, Harvard University, Massachusetts Institute of Technology (MIT), Princeton University y University of Texas, Austin, entre otras.

Recibido: 11-08-2015

Aprobado: 14-02-2016 
